\title{
Multiplicative Spatio-Temporal Models For Remotely Sensed Normalized Difference Vegetation Index Data
}

Nicolle Clements, Saint Joseph's University, USA

Sanat Sarkar, Temple University, USA

William Wei, Temple University, USA

\begin{abstract}
Vegetation forecasting is closely tied to many important international concerns, including: monitoring the impacts of global climate change and energy usage, managing the consumption of natural resources, predicting the spread of invasive species, and protecting endangered species. In light of these issues, this article develops vegetation forecasting models for normalized difference vegetation index (NDVI) data recorded by remote sensing via satellites in East Africa. Spatio-temporal auto-regressive moving average (STARMA) is a class of models that can be used in monitoring and forecasting, but it must be modified for highly seasonal processes with temporal trends. We propose to use multiplicative STARMA models to estimate and forecast NDVI values for sub-regions that have previously been detected to have statistically significant temporal trends. For illustration, we select a few East African sub-region's NDVI series to apply the proposed models and demonstrate the advantages over traditional modeling techniques.
\end{abstract}

Keywords: East Africa; NDVI; Spatio-Temporal; STARMA

\section{INTRODUCTION}

nalysis of vegetation life cycles is fundamental in monitoring and planning agricultural endeavors.
In particular, gaining knowledge of current vegetation trends and using them to make accurate
predictions is essential to minimize times of food scarcity and manage the consumption of natural resources in underdeveloped countries.

For this reason, there are many agencies and organizations that focus on the study of land use and land cover trends, linking them to climate change and the socioeconomic consequences of these changes. The United States Global Change Research Program (Land Use and Land Cover Change Interagency Working Group), the United Nations Framework Convention on Climate Change (Land Use, Land Use Change, and Forestry), and NASA's Land Cover Land Use Change Program are just three examples of well-known interdisciplinary/ interagency programs that conduct and sponsor research related to the question of global land change. The United Nations has given recent attention to precipitation and vegetation monitoring in Eastern Africa, where a severe drought hit the entire region in mid-2011. The drought has caused a food crisis across Somalia, Ethiopia, and Kenya, threatening the livelihood of over 10 million people.

To help avoid such crises, data collection on vegetation and land cover are typically done through satellite remote sensing. The remote sensing imagery is used to convert the observed elements (i.e., the image color, texture, tone, and pattern) into numeric quantities at each pixel in the image. The image pixels correspond to a square grid of land, the size of which depends on the satellite's resolution.

One such numeric value is the normalized difference vegetation index (NDVI). The NDVI has been shown to be highly correlated with vegetation parameters such as green-leaf biomass and green-leaf area and hence is of considerable value for vegetation monitoring (Curran, 1980; Jackson, Slate, \& Pinter, 1983). The NDVI standard 
scale ranges from -1 to 1 , indicating how much live green vegetation is contained in the targeted pixel. An NDVI value close to 1 indicates more abundant vegetation. Low values of NDVI (say 0.1 and below) correspond to scarce vegetation consisting mostly of rock, sand, and bare soil, for example. A range of moderate values (0.2 to 0.3$)$ indicates short vegetations such as shrub or grassland; larger NDVI values can be found in rainforests (0.6 to 0.8).

Vegetation activity is a continuous space-time process and NDVI data provide a space-time lattice system, in the sense that observations are available over equally spaced regular grids. Often, the spatial resolution ranges from 1 to 8 kilometers, while the temporal one ranges from 7 days to 1 month.

Time series models are tempting for representing such processes. Univariate autoregressive integrated moving average (ARIMA) models are widely utilized for a single time series, such as the modeling of one pixel's NDVI series. Univariate modeling can be extended to incorporate the influence of one or more external factors, such as Intervention Analysis, when there is a known external factor within the ARIMA model or Transfer function models that combine information of other related time series to model the underlying disturbance or relationship.

However, in the present application where the NDVI series in neighboring pixels are likely to be correlated, it is more appropriate to consider modeling several pixels jointly to understand the structure of the NDVI values over both time and space. To do so, space-time auto-regressive (STAR) models or space-time auto-regressive moving average (STARMA) models can be used. STAR and STARMA are a class of suitable linear models where each observation is placed in linear relationship with previous and surrounding values and errors to produce forecasts.

These models, however, are limited by stationarity and thus must be adapted to possible non-stationarity (e.g. temporal trends) and to include their seasonal patterns, which has yet to be done in recent studies. This article proposes to use multiplicative vector STARMA models for sub-regions of lattice data that have previously been detected to have statistically significant temporal trends. The temporal trends will be represented by a deterministic trend term in the model and the seasonal patterns will be modeled by multiplicative seasonal auto-regressive or seasonal moving average components, if needed, after seasonal differencing. Our aim is to show that these multiplicative STARMA models can forecast NDVI more accurately than traditional ARIMA modeling techniques, and thus provide greater knowledge to such land monitoring agencies.

\section{DATA DESCRIPTION}

East Africa spans a wide variety of climate types and precipitation regimes which is reflected in its vegetation cover. To capture this, satellite imagery was collected over a sub-Saharan region of East Africa that includes five countries in their entirety (Kenya, Uganda, Tanzania, Burundi, and Rwanda) and portions of seven countries (Somalia, Ethiopia, Sudan, Democratic Republic of Congo, Malawi, Mozambique, and Zimbabwe). This roughly 'rectangular' region extends from $27.8^{\circ} \mathrm{E}$ to $42.0^{\circ} \mathrm{E}$ longitude and $15^{\circ} \mathrm{S}$ to $6.2^{\circ} \mathrm{N}$ latitude. Also included in the region are several large East African Great Lakes such as Lake Victoria, Lake Malawi, and Lake Tanganyika.

The remotely sensed images were recorded twice a month between 1982 and 2006 and then converted to NDVI values. Hence, the spatio-temporal data set consists of approximately 50,000 sites (pixels), each with 600 time series observations ( 24 observations per year over 25 years). The satellite's resolution corresponds to each pixel spanning an $8 \mathrm{~km} \times 8 \mathrm{~km}$ grid of land. This Global Inventory Modeling and Mapping Studies (GIMMS) data set is derived from imagery obtained from the Advanced Very High Resolution Radiometer (AVHRR) instrument onboard the NOAA satellite series 7, 9, 11, 14, 16 and 17. The NDVI values have been corrected for calibration, view geometry, volcanic aerosols, and other effects not related to vegetation change (Tucker et. al., 2005).

Figure 1(A) displays the periodicity, or periodic pattern, which is the cyclic/seasonal behavior of the NDVI values. For example, some regions have a unimodal periodic pattern each year (periodicity of 12 months) while other regions have a bimodal pattern (periodicity of 6 months). Figure 1(B) displays the average NDVI values for each grid point (site) over the region. Blue areas indicate regions containing only water (Indian Ocean, Lake Victoria, etc.), and thus no vegetation index was recorded. The light and dark green areas have more green vegetation, on average, compared to the drier areas, represented with yellow, orange, and red. In this figure, one can 
see how this East African region spans the NDVI scale. Desert regions (with low NDVI) are within a few hundred kilometers of wetlands and rain forests (with extremely high NDVI), illustrating the large variability of climate types and precipitation regimes in this region.

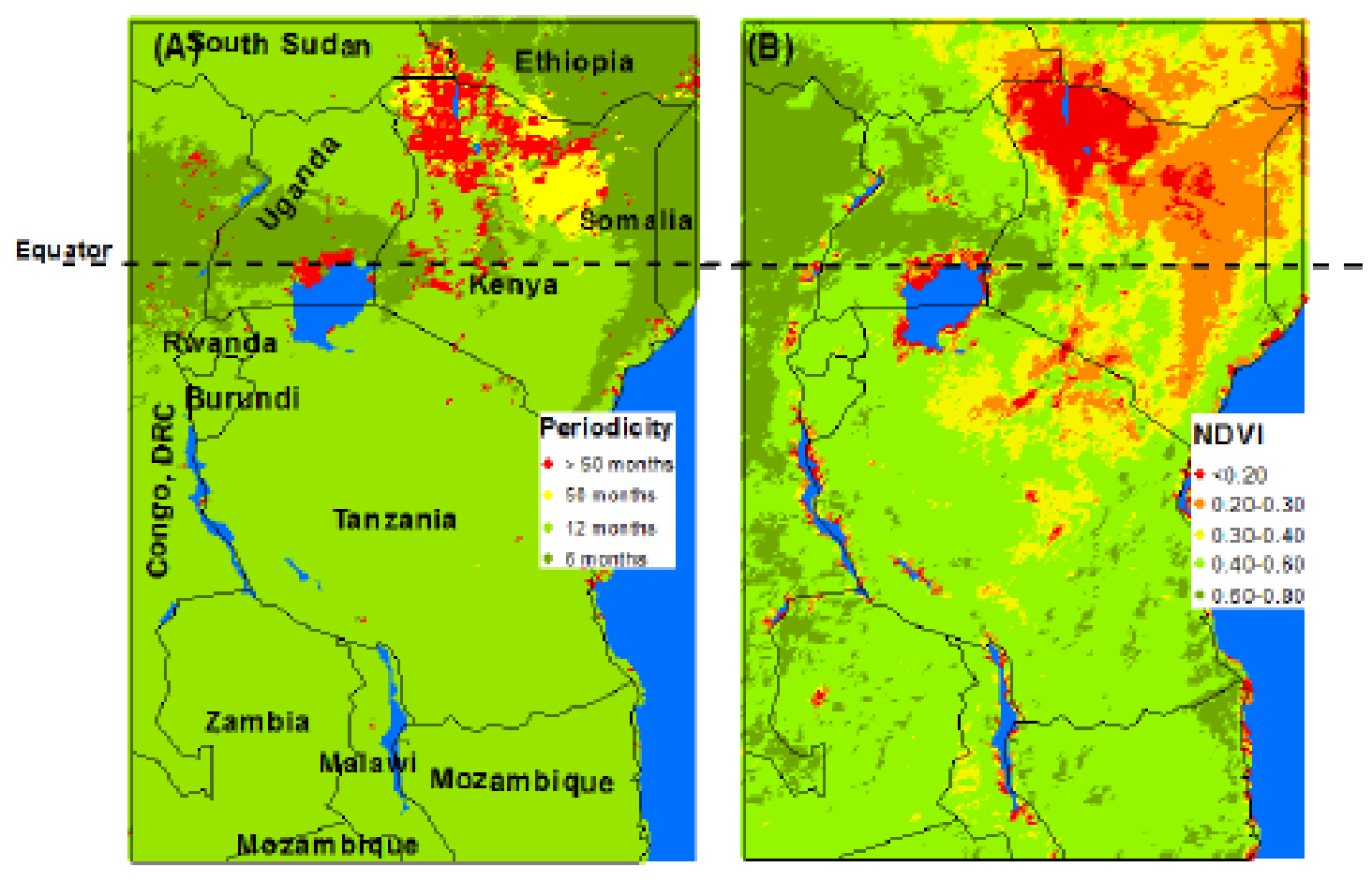

Figure 1 (A): Periodicity Categories Of The NDVI Time Series For The Time Period From 1982-2006

Figure 1(B): Average NDVI For The Time Period Of 1982-2006 In The East Africa Region

We consolidated all the negative NDVI values to zero, as commonly done in vegetation monitoring, and rescaled the remaining values by 1,000. Negative NDVI values indicate non-vegetation areas, and so they are of no use in our statistical analysis. Prior to the analysis, we examined the data for quality assurance and eliminated a small number of pixels that were found to have several consecutive years with identical data values, which may be due to data entry errors or machine malfunction.

Vegetative analysis in this region is of interest for a variety of reasons, including the importance of the region for global biodiversity and the vulnerability of the region to climate change, deforestation of the Congo, urban expansion, civil unrest, and agricultural practices. As a result, there have been several studies in this region using various multi-temporal remote sensing techniques (Pelkey, 2000; Pelkey, 2003; Davenport, 1993; Vrieling, deBeurs, \& Brown, 2008). The objective of this paper is to provide an additional approach to modeling and forecasting NDVI in this region using spatio-temporal auto-regressive moving average models and discuss some advantages of this class of models over traditional techniques.

\section{MULTIPLICATIVE STARMA MODELS}

Before diving into the modeling and forecasting of this large dataset, one must select which pixels are of interest for modeling. It would be too labor intensive to individually model the 50,000 univariate time series. On the other hand, using one multivariate time series model of dimension 50,000 is also infeasible due to an enormous number of parameters to estimate ( $p \gg n$ problem). Thus, one must utilize a screening process to select the pixels or sub-regions of pixels that are of interest for further investigation. In this application, where detecting increasing or decreasing vegetation trends is valuable for land use managers, researchers might want to select pixels with such trends for further modeling. 
Clements (2013) has tackled this problem from a multiple testing point of view. They suggest selecting the pixels warranting modeling investigation by testing each pixel's NDVI seasonal series for temporal trends and combining the resulting $p$-values in a multiple testing procedure designed to control an overall measure of mixed directional and non-directional false discoveries. Utilizing the information gleaned after applying this procedure, more sophisticated modeling techniques (i.e., STARMA models) can be applied to the sub-regions detected to have temporal trends. By first detecting pixels having trends using such multiple testing procedures, we are protected from investigating too many falsely discovered locations.

In the subsequent subsections $(3.1,3.2$, and 3.3), we describe the steps to identify and estimate parameters in STARMA models. In Section 4, we apply these steps to two sub-regions in the East African NDVI dataset that were screened by the procedure suggested in Clements (2013) to have significant temporal trends and compare the results to ARIMA models in Section 5.

\subsection{Notation And Background}

First, we shall introduce basic notation and background information about STARMA models and how they pertain to the East African vegetation application. If one thinks of the entire dataset, denoted as $\mathbf{Z}$, organized as a three-way matrix, then $\mathbf{Z}=\left\{Z_{i, j, t}\right\}$ is $290 \times 196 \times 600$, where $i, j, t$ indicate latitude, longitude, and time, respectively. Taking the approach based on Tiao and Box (1981), a simple example of a first-order dependence in time and second order dependence in space STAR model for $Z_{i, j, t}$ is:

$$
\begin{aligned}
Z_{i, j, t}= & \mu_{i, j}+\phi_{1} Z_{i, j, t-1}+\phi_{2} Z_{i-1, j-1, t-1}+\phi_{3} Z_{i-1, j, t-1}+ \\
& \ldots+\phi_{9} Z_{i+1, j+1, t-1}+a_{i, j, t} \\
= & \mu+\phi^{\prime} x_{i, j, t}+a_{i, j, s},
\end{aligned}
$$

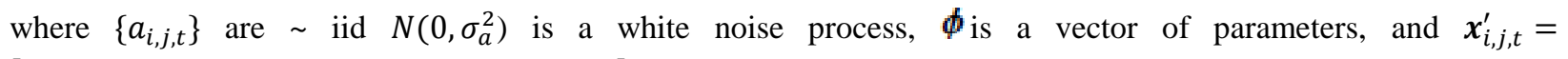
$\left[Z_{i, j, t-1}, Z_{i-1, j-1, t-1}, Z_{i-1, j, t-1}, \ldots, Z_{i+1, j+1, t-1}\right]$ is the vector of 'regressors'.

To define a more general STARMA model, let the order of space dependency be denoted by $S$, the autoregressive order by $p$, and the moving average order by $q$. The $\operatorname{STARMA}(S, p, q)$ model for $Z_{i, j, t}$ takes the form:

$$
\begin{aligned}
Z_{i, j, t}=\mu_{i, j} & +\sum_{l=1}^{p} \sum_{g=-S}^{S} \sum_{h=-S}^{S}\left\{\phi_{g h l} Z_{i-g, j-h, t-l}\right\}+ \\
& \sum_{m=1}^{q} \sum_{g=-S}^{S} \sum_{h=-S}^{S}\left\{\theta_{g h m} a_{i-g, j-h, t-m}\right\}+a_{i, j, s}
\end{aligned}
$$

We consider 'stacking' the series of the neighboring pixels to form a $(2 S+1)^{2}$-dimensional time series vector, say $\boldsymbol{Z}_{t}$. By letting $K=(2 S+1)^{2}$, the dimension of the vector, for convenience, we can write the model in matrix notation and model the series jointly. Using the backshift operator $B$ to define $B Z_{i, j, t}=Z_{i, j, t-1}$, we can write $\operatorname{STARMA}(S, p, q)$ as 


$$
\phi(B) \mathbf{Z}_{t}=\mu+\theta(B) \mathbf{a}_{t}
$$

Where

$$
\begin{aligned}
& \phi(B)=I-\phi_{1} B-\cdots-\phi_{p} B^{p} \\
& \boldsymbol{\theta}(B)=I-\boldsymbol{\theta}_{1} B-\cdots-\boldsymbol{\theta}_{q} B^{q}
\end{aligned}
$$

are the matrix polynomials in $B$, the $\boldsymbol{\phi}$ 's and $\boldsymbol{\theta}$ 's are $K \times K$ matrices, $\boldsymbol{\mu}$ is a $K \times 1$ constant vector, and $\boldsymbol{a}_{t}$ is a sequence of $K \times 1$ random shock vectors identically and independently distributed as a normal distribution with zero mean and covariance matrix $\boldsymbol{\Sigma}$.

For example, consider the model of order $(S, p, q)=(1,1,1)$ with $\boldsymbol{\mu}=0$. The model reduces to

$$
\begin{aligned}
& \left(\left[\begin{array}{lllll}
1 & 0 & 0 & 0 & 0 \\
0 & 1 & 0 & 0 & 0 \\
0 & 0 & 1 & 0 & 0 \\
0 & 0 & 0 & 1 & 0 \\
0 & 0 & 0 & 0 & 1
\end{array}\right]-\left[\begin{array}{lllll}
\phi_{11} & \phi_{12} & \phi_{13} & \phi_{14} & \phi_{15} \\
\phi_{21} & \phi_{22} & \phi_{23} & \phi_{24} & \phi_{25} \\
\phi_{31} & \phi_{32} & \phi_{33} & \phi_{34} & \phi_{35} \\
\phi_{41} & \phi_{42} & \phi_{43} & \phi_{44} & \phi_{45} \\
\phi_{51} & \phi_{52} & \phi_{53} & \phi_{54} & \phi_{55}
\end{array}\right]\right)\left[\begin{array}{l}
Z_{1, s} \\
Z_{2, s} \\
Z_{3, s} \\
Z_{4, s} \\
Z_{5, s}
\end{array}\right] \\
= & \left(\left[\begin{array}{lllll}
1 & 0 & 0 & 0 & 0 \\
0 & 1 & 0 & 0 & 0 \\
0 & 0 & 1 & 0 & 0 \\
0 & 0 & 0 & 1 & 0 \\
0 & 0 & 0 & 0 & 1
\end{array}\right]-\left[\begin{array}{lllll}
\theta_{11} & \theta_{12} & \theta_{13} & \theta_{14} & \theta_{15} \\
\theta_{21} & \theta_{22} & \theta_{23} & \theta_{24} & \theta_{25} \\
\theta_{31} & \theta_{32} & \theta_{33} & \theta_{34} & \theta_{35} \\
\theta_{41} & \theta_{42} & \theta_{43} & \theta_{44} & \theta_{45} \\
\theta_{51} & \theta_{52} & \theta_{53} & \theta_{54} & \theta_{55}
\end{array}\right]\right)\left[\begin{array}{l}
B \\
a_{1, s} \\
a_{2, s} \\
a_{3, s} \\
a_{4, s} \\
a_{5, s}
\end{array}\right] .
\end{aligned}
$$

In this model, the diagonal elements of the parameter matrices indicate how an individual series is affected by its own past data (temporal dependency). The off-diagonal elements in each lag matrix represent the influence of one series on the other series (spatial dependency).

Often in environmental data applications, the series will have a strong seasonal pattern that can dominate the model. These patterns can be accounted for in the model by taking seasonal differences; for instance, if $D$ is the length of the seasonal pattern, then $\left(\boldsymbol{Z}_{t}-\boldsymbol{Z}_{t-D}\right)=\left(1-B^{D}\right) \boldsymbol{Z}_{t}$. For example, $D=4$ for quarterly data, $D=12$ for monthly data, etc. Additionally, multiplicative seasonal auto-regressive and seasonal moving average terms may be needed in the model. For convenience, we will denote $\boldsymbol{\phi}_{p}(B)$ and $\boldsymbol{\theta}_{q}(B)$ as the regular auto-regressive and moving average factors and $\boldsymbol{\Phi}_{P}\left(B^{D}\right)$ and $\boldsymbol{\Theta}_{Q}\left(B^{D}\right)$ as the seasonal auto-regressive and moving average factors of order $P$ and $Q$, respectively. This leads to a multiplicative $\operatorname{STARMA}(S, p, q) \times(P, Q)_{D}$ model of the form: 


$$
\Phi_{P}\left(B^{D}\right) \phi_{p}(B)\left(1-B^{D}\right) \mathbf{Z}_{t}=\mu+\boldsymbol{\theta}_{q}(B) \Theta_{Q}\left(B^{D}\right) \mathbf{a}_{t}
$$

where

$$
\begin{aligned}
& \Phi_{P}\left(B^{D}\right)=I-\Phi_{1} B^{D}-\Phi_{2} B^{2 D}-\cdots-\Phi_{P} B^{P D} \\
& \Theta_{Q}\left(B^{D}\right)=I-\Theta_{1} B^{D}-\Theta_{2} B^{2 D}-\cdots-\Theta_{Q} B^{Q D}
\end{aligned}
$$

and $\boldsymbol{a}_{t}$ 's are $K$-dimensional white noise random vectors with zero mean and covariance matrix structure:

$$
\operatorname{Cov}\left[\mathbf{a}_{t}, \mathbf{a}_{t+k}\right]=E\left[\mathbf{a}_{t} \mathbf{a}_{t+k}^{\prime}\right]=\left\{\begin{array}{cc}
\mathbf{\Sigma} & k=0 \\
0 & k \neq 0
\end{array}\right.
$$

where $\boldsymbol{\Sigma}$ is a $K \times K$ symmetric positive definite matrix. In general, for a series involving differencing of order $D$ (i.e., $\left(1-B^{D}\right) \boldsymbol{Z}_{t}$ ), Wei (2006) shows the non-zero constant $\boldsymbol{\mu}$ corresponds to the $D^{\text {th }}$ derivative coefficient of the deterministic trend model

$$
Z_{1}=\alpha_{0}+\alpha_{1} t+\ldots+\alpha_{D} t^{D}
$$

When differencing is needed in the model $(D>0), \boldsymbol{\mu}$ is known as the deterministic trend term and can dominate the series, forcing it to follow a deterministic pattern. Thus, $\boldsymbol{\mu}$ is usually assumed to be zero, unless it is clear from the data that a deterministic component is really needed.

In the case of NDVI modeling and forecasting, the deterministic trend term is indeed needed for the pixels selected from the screening process in Clements (2013). The nonstationarity in the mean NDVI is expected for these selected pixels since they were detected to have a significant increasing/decreasing trend pattern in the seasonal averages using a multiple testing procedure designed to control an overall measure of mixed directional and nondirectional false discoveries prior to the modeling.

\subsection{Model Identification}

Similar to univariate time series, to determine the order $(p, q)$ and $(P, Q)$ in the $\operatorname{STARMA}(S, p, q) \times(P, Q)_{D}$ model, the cross covariance and cross correlation matrices (CMM) are employed. For a stationary vector time series $\boldsymbol{Z}_{t}$, the lag $l$ cross covariance matrix is defined as

$$
\Gamma(l)=E\left[\left(\mathbf{Z}_{t}-\boldsymbol{\mu}\right)\left(\mathbf{Z}_{t+l}-\boldsymbol{\mu}\right)^{\prime}\right]=\left\{\gamma_{i, j}(l)\right\}, \text { for } l=0,1,2, \ldots \text { and } i, j=1,2, \ldots, K
$$

The corresponding cross correlation matrix (CCM) is $\boldsymbol{\rho}(l)=\boldsymbol{V}^{-1} \boldsymbol{\Gamma}(l) \boldsymbol{V}^{-1}$, where $\boldsymbol{V}$ is a diagonal matrix containing $\gamma_{i, i}(0)^{1 / 2}$ as its $i^{\text {th }}$ diagonal element. The $(i, j)$ element of the lag $l$ matrix is an indication of how series $i$ is influenced by series $j$ at lag $l$. The cross covariance and correlation matrices can be estimated from the data by $C_{i, j}(l)=\frac{1}{n} \sum_{t=l+1}^{n}\left(Z_{i, t}-\bar{Z}_{i}\right)\left(Z_{j, t+1}-\bar{Z}_{j}\right)$ and $\hat{\rho}_{i, j}(l)=C_{i, j}(l) /\left[C_{i, i}(0) C_{j, j}(0)\right]^{1 / 2}$, respectively, where $\bar{Z}_{i}$ and $\bar{Z}_{j}$ are the means for the $i^{t h}$ and $j^{t h}$ series. The cross correlation matrices are interpreted parallel to the univariate ACF, where an AR model is indicated by a 'die off' pattern and a MA model will 'cut off' after a particular lag.

When a vector time series has both AR and MA components, the model cannot be easily identified. Tiao and Tsay (1983) introduced the extended cross correlation matrices (ECCM) method, a multivariate analogue to the 
EACF in univariate modeling. The ECCM does well to identify the order $(p, q)$ and $(P, Q)$ for STARMA models when the number of series, $K=(2 S+1)^{2}$, is small (e.g. one to five series). The ECCM gives strongest consideration to lower order models, since the number of parameters to estimate can be quite large. The general $\operatorname{STARMA}(S, p, q) \times(P, Q)_{D}$ model contains $K^{2}(p+q+P+Q)+K$ parameters (excluding the covariance matrix, $\boldsymbol{\Sigma}$ ).

\subsection{Estimating Parameters}

After the STARMA model is tentatively identified using ECCM, the constant vector $\boldsymbol{\mu}$, matrices $\boldsymbol{\phi}_{i}(i=$ $1,2, \ldots, p), \boldsymbol{\theta}_{j}(j=1,2, \ldots, q), \boldsymbol{\Phi}_{i}(i=1,2, \ldots, P)$, and $\boldsymbol{\Theta}_{j}(j=1,2, \ldots, Q)$, and the covariance matrix $\boldsymbol{\Sigma}$, can be estimated by maximizing the corresponding likelihood function.

Two useful simplifying approximations to the likelihood function are the 'conditional' likelihood function, proposed in Wilson (1973), and the 'exact' likelihood function, proposed in Hillmer and Tiao (1979). The exact approach is more computationally intensive, but it can drastically reduce the biases in estimating the moving average coefficients, especially when some of the roots are near the unit circle.

Once the parameters are estimated, the next step is diagnostic checking of the residuals to determine the adequacy of the model fit and to search for potential improvements. In multivariate models, diagnostic checks can include plotting the residuals and examining the cross correlation matrices (CCM) to determine if $\boldsymbol{a}_{t}$ is a white noise process. If the model is adequate, it can be used to forecast the series for future lags.

\section{EXAMPLES OF STARMA MODELS USING EAST AFRICAN NDVI}

In this section, we build multiplicative STARMA models for selected NDVI series. Since the number of parameters can be too large to estimate in higher ordered models, we seek a parsimonious model, on which to interpret parameters and do short-term forecasting. We select East African sub-regions that have been found to have significant vegetation temporal trends using a multiple testing procedure designed to control an overall measure of mixed directional and nondirectional false discoveries (Clements, 2013). This screening procedure operated by grouping the pixels into suitable sub-regions, the size of which were estimated through a semivariogram, to capture local spatial dependency through the so-called grouped $p$-values. Thus, we follow a similar approach in selecting the spatial dependency parameter $S=1$ and form $K=5$ dimensional STARMA models. The following two examples of modeling and forecasting were done using the SCA Statistical System, version 8.1.

\subsection{Modeling And Forecasting For Terkaka, South Sudan}

For the first example, a selected city, Terkaka, South Sudan (coordinates $31.01861^{\circ} \mathrm{E}, 5.664367^{\circ} \mathrm{N}$ ), was detected to have a significant increasing temporal trend in the NDVI values. It would be useful to use this knowledge to do future vegetation forecasting and account for the spatial dependency of neighboring pixels. Figure 2, below, shows the time series observations for Terkaka, South Sudan, with increasing trend line (top) and the series of the neighboring pixels (bottom). One can see that the NDVI values of neighboring pixels have a similar repetitive pattern every 24 lags, which is expected due to the data collection occurring twice per month. 
NDVI Series for Terkaka, South Sudan

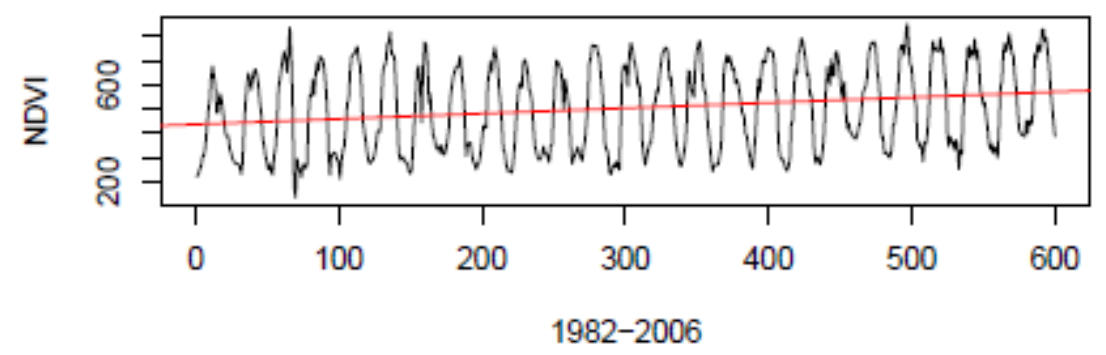

NDVI Series for 5 Neighboring Pixels

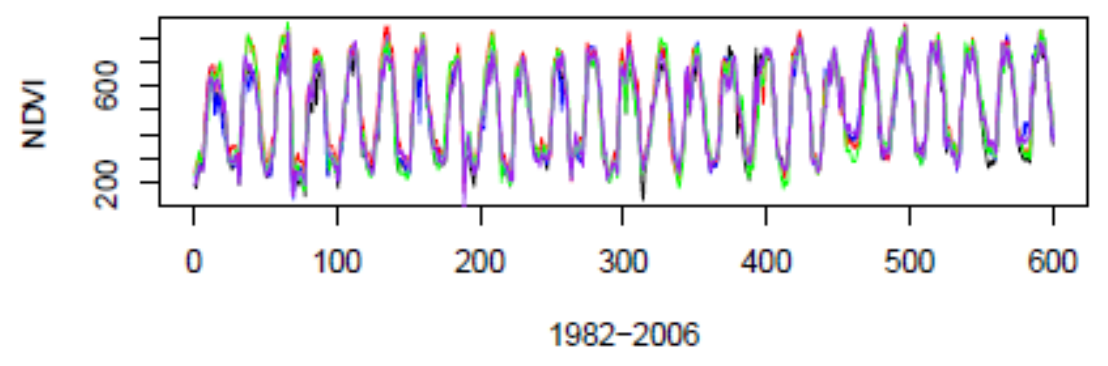

Figure 2: Terkaka's NDVI Series (Top) And With Neighboring Pixels (Bottom)

After careful examination of the ECCM table, ACF, and PACF plots of all five pixel's NDVI series, the tentative model selected is $\operatorname{STARMA}(S=1, p=2, q=1) \times(P=0, Q=1)$ with $D=24$ :

$$
\left(\mathbf{I}-\phi_{1} B-\phi_{2} B^{2}\right)\left(\mathbf{I}-\operatorname{Diag}\left(B^{24}\right)\right) Z_{t}=\mu+\left(\mathbf{I}-\theta_{1} B\right)\left(\mathbf{I}-\Theta_{24} B^{24}\right) \mathbf{a}_{t},
$$

where $\operatorname{Diag}\left(B^{24}\right)$ is a $5 \times 5$ diagonal matrix with diagonal elements $B^{24}$, indicating the need for differencing from the repetitive seasonal pattern every 24 lags. Using the 'exact' likelihood approach to estimate the parameters, the resulting matrices are: 


$$
\begin{aligned}
& \boldsymbol{\mu}=\left[\begin{array}{c}
0 \\
3.22 \\
0 \\
0 \\
0
\end{array}\right], \boldsymbol{\phi}_{1}=\left[\begin{array}{ccccc}
.455 & .381 & 2.715 & -.912 & -1.645 \\
-.380 & 1.219 & 2.771 & -1.267 & -1.283 \\
.265 & .241 & 2.306 & -.556 & -1.197 \\
-.603 & .976 & 3.135 & -.751 & -1.508 \\
.454 & -.373 & 1.088 & -.267 & 0
\end{array}\right], \phi_{2}=\left[\begin{array}{ccccc}
.187 & -.534 & -1.607 & .642 & .909 \\
.301 & -.608 & -1.670 & .931 & .662 \\
-.139 & -.337 & -.965 & .371 & .636 \\
.465 & -.927 & -1.925 & 1.015 & .768 \\
-.259 & .112 & -.556 & .154 & .219
\end{array}\right] \text {, } \\
& \boldsymbol{\theta}_{1}=\left[\begin{array}{ccccc}
-.139 & .213 & 2.627 & -.868 & -1.701 \\
-.287 & .445 & 2.634 & -1.115 & -1.457 \\
.348 & .134 & 1.583 & -.549 & -1.310 \\
-.489 & .902 & 3.043 & -1.458 & -1.613 \\
.446 & -.363 & .987 & -.254 & -.760
\end{array}\right], \Theta_{24}=\left[\begin{array}{ccccc}
.868 & 0 & 0 & 0 & 0 \\
0 & .764 & 0 & 0 & 0 \\
0 & 0 & .835 & 0 & 0 \\
0 & 0 & 0 & .809 & 0 \\
0 & 0 & 0 & 0 & .895
\end{array}\right] \text {, and } \\
& \Sigma=\left[\begin{array}{lllll}
2318.92 & 1652.48 & 1475.09 & 1255.38 & 1697.02 \\
1652.48 & 2183.63 & 1491.65 & 1218.73 & 1511.44 \\
1475.09 & 1491.65 & 2245.01 & 1647.27 & 1635.91 \\
1255.38 & 1218.73 & 1647.27 & 2069.41 & 1315.84 \\
1697.02 & 1511.44 & 1635.91 & 1315.84 & 2320.32
\end{array}\right]
\end{aligned}
$$

The residuals of this model indicate a white noise process, validating the adequacy of the model fit. The pixel having significant increasing NDVI trends (Terkaka, South Sudan) is $\boldsymbol{Z}_{2, t}$ of the model. Writing the forecasting equation for this pixel is:

$$
\begin{aligned}
& Z_{2, t+1}=Z_{2, t-23}+3.22-0.38 Z_{1, t}+1.219 Z_{2, t}+2.771 Z_{3, t}-1.267 Z_{4, t}-1.283 Z_{5, t}+0.301 Z_{1, t-1}-0.608 Z_{2, t-1}-1.67 Z_{3, t-1}+ \\
& 0.931 Z_{4, t-1}+0.662 Z_{5, t-1}+0.38 Z_{1, t-24}-1.219 Z_{2, t-24}-2.771 Z_{3, t-25}+1.267 Z_{4, t-25}+1.283 Z_{5, t-24}-0.301 Z_{1, t-25}+ \\
& 0.608 Z_{2, t-25}+1.67 Z_{3, t-24}-0.931 Z_{4,-24}-0.662 Z_{5, t-25}+0.287 a_{1, t}-0.445 a_{2, t}-2.634 a_{3, t}+1.115 a_{4, t}+1.457 a_{5, t}- \\
& 0.764 a_{2, t-23}-0.249 a_{1, t-24}+0.339 a_{2, t-24}+2.199 a_{3, t-24}-0.902 a_{4, t-24}-1.304 a_{5, t-24}
\end{aligned}
$$

By examining the first two terms of the forecast equation, one can see that the forecast for time $t+1$ takes the NDVI value from time $t-23$ (the previous year's value at that time of month) and adds the deterministic trend constant 3.22, which is significantly different from zero $(p=0.001)$. As discussed in Section 3.1, deterministic trend terms should be used with caution since they can dominate the series. However, it is important to include it here since Terkaka's NDVI series was previously detected to have a significant increasing trend; thus, it is not surprising that $\mu_{2}$ is significant in this model. The value $\mu_{2}=3.22$ indicates that, from year-to-year, the NDVI values are increasing by 3.22 , on average.

The remaining terms in the forecasting equation can be attributed to the autoregressive and moving average components' past lags of Terkaka $\left(\boldsymbol{Z}_{2, t}\right)$ and its neighbors $\left(\boldsymbol{Z}_{1, t}, \boldsymbol{Z}_{3, t}, \boldsymbol{Z}_{4, t}, \boldsymbol{Z}_{5, t}\right)$. Out of the four neighbors, the series $\boldsymbol{Z}_{3, t}$ is more dominant than $\boldsymbol{Z}_{1, t}, \boldsymbol{Z}_{4, t}, \boldsymbol{Z}_{5, t}$, as seen by the magnitude of the AR and MA coefficients. 
This model's forecast for one year (24 lags) ahead, with the 95\% bands, is displayed in Figure 3.

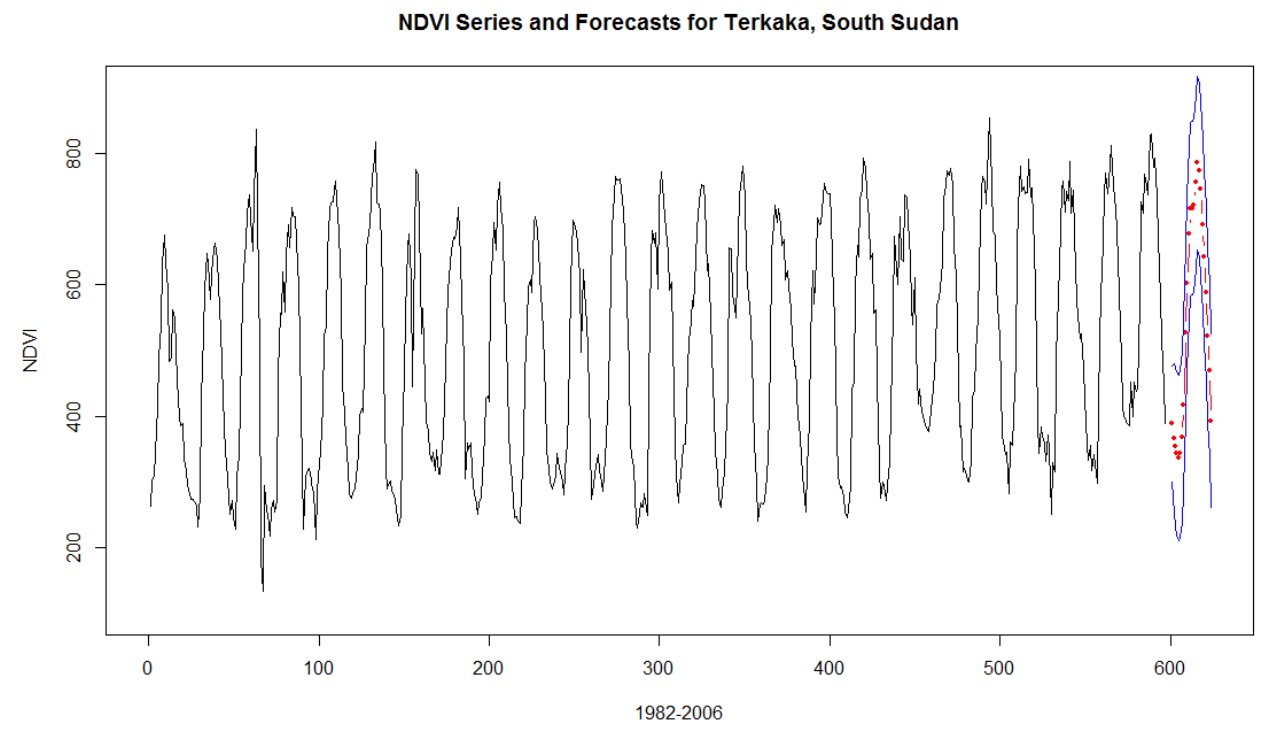

Figure 3: Terkaka's NDVI Series With One Year Forecasts And Confidence Bands

\subsection{Modeling And Forecasting For Kilosa, Tanzania}

The second example selected was Kilosa, Tanzania (coordinates $36.98224^{\circ} \mathrm{E}, 6.553814^{\circ} \mathrm{S}$ ). This pixel was detected to have significant decreasing temporal trends in the seasonal NDVI averages. Figure 4, below, shows the time series observations for Kilosa, Tanzania, with a decreasing trend line (top) and the series of the neighboring pixels (bottom).
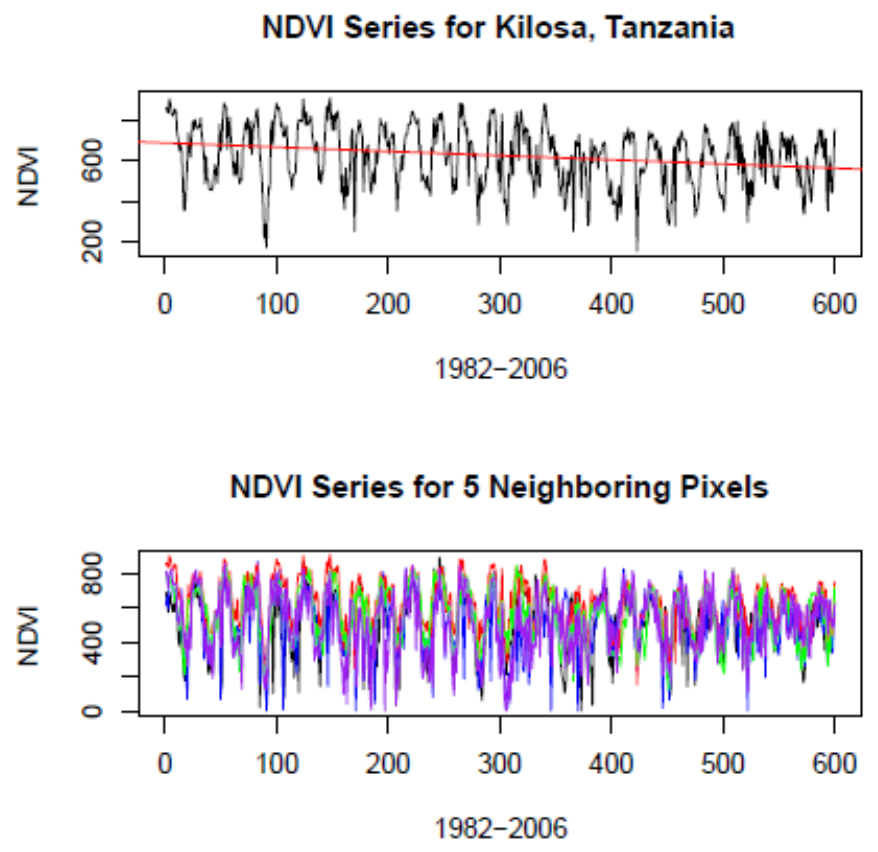

Figure 4: Kilosa's NDVI Series (Top) And With Neighboring Pixels (Bottom) 
After careful examination of the ECCM table, ACF, and PACF plots of all five pixel's NDVI series, the tentative model selected is also STARMA $(1,2,1) \times(0,1)$ with $D=24$

$$
\left(\mathbf{I}-\phi_{1} B-\phi_{2} B^{2}\right)\left(\mathbf{I}-\operatorname{Diag}\left(B^{24}\right)\right) \mathbf{Z}_{t}=\boldsymbol{\mu}+\left(\mathbf{I}-\boldsymbol{\theta}_{1} B\right)\left(\mathbf{I}-\mathbf{\Theta}_{24}\right) \mathbf{a}_{t},
$$

where $\operatorname{Diag}\left(B^{24}\right)$ is a $5 \times 5$ diagonal matrix with diagonal elements $B^{24}$, indicating the repetitive seasonal pattern every 24 lags. Using the 'exact' likelihood approach to estimate the parameters, the resulting matrices are:

$$
\begin{aligned}
& \mu=\left[\begin{array}{c}
0 \\
0 \\
-3.301 \\
0 \\
0
\end{array}\right], \phi_{1}=\left[\begin{array}{ccccc}
1.362 & -.384 & -.607 & 1.343 & 1.918 \\
0 & .875 & .344 & 1.567 & 1.052 \\
.239 & -.483 & 0 & .101 & .665 \\
-.270 & .389 & -.209 & .530 & -.905 \\
-.451 & .464 & 1.067 & .476 & .367
\end{array}\right], \phi_{2}=\left[\begin{array}{ccccc}
-.290 & 0 & .304 & -.765 & -.845 \\
0 & -.313 & -.175 & -1.012 & -.316 \\
0 & .153 & .184 & 0 & -.314 \\
0 & 0 & 0 & .154 & .323 \\
.161 & -.133 & -.548 & -.301 & .103
\end{array}\right] \text {, } \\
& \theta_{1}=\left[\begin{array}{ccccc}
.962 & -.461 & -.640 & 1.507 & 1.935 \\
0 & .287 & .339 & 1.603 & 1.087 \\
.214 & -.426 & -.431 & .106 & .671 \\
-.266 & .484 & -.229 & 0 & -.881 \\
-.443 & .315 & 1.131 & .404 & 0
\end{array}\right], \Theta_{24}=\left[\begin{array}{ccccc}
.933 & 0 & 0 & 0 & 0 \\
0 & .824 & 0 & 0 & 0 \\
0 & 0 & .888 & 0 & 0 \\
0 & 0 & 0 & .787 & 0 \\
0 & 0 & 0 & 0 & .846
\end{array}\right] \text {, and } \\
& \mathbf{\Sigma}=\left[\begin{array}{lllll}
15188.1 & 7485.28 & 5121.68 & 3752.67 & 7714.98 \\
7485.28 & 18020.9 & 5279.92 & 3210.38 & 110715 \\
5121.68 & 5279.92 & 8187.59 & 4829.22 & 7092.84 \\
3752.67 & 3210.38 & 4829.22 & 5992.50 & 4675.01 \\
7714.98 & 110715 & 7092.84 & 4675.01 & 7858.60
\end{array}\right]
\end{aligned}
$$

The residuals of this model indicate a white noise process, validating the adequacy of the fit. The pixel, having significant decreasing NDVI trends (Kilosa, Tanzania), is $\boldsymbol{Z}_{3, t}$ of the model. Writing the forecasting equation for this pixel is:

$$
\begin{gathered}
Z_{3, t+1}=Z_{3, t-23}-3.301+0.239 Z_{1, t}-0.483 Z_{2, t}+0.101 Z_{4, t}+0.665 Z_{5, t}+0.153 Z_{2, t-1}+0.184 Z_{3, t-1}-0.314 Z_{5, t-1}- \\
0.239 Z_{1, t-24}+0.483 Z_{2, t-24}-0.101 Z_{4, t-24}-0.665 Z_{5, t-24}-0.153 Z_{2, t-25}-0.184 Z_{3, t-25}+0.314 Z_{5, t-25}-0.214 a_{1, t}+0.426 a_{2, t}+ \\
0.431 a_{3, t}-0.106 a_{4, t}-0.671 a_{5, t}-0.888 a_{3, t-23}+0.199 a_{1, t-24}-0.351 a_{2, t-24}-0.383 a_{3, t-24}+0.083 a_{4, t-24}+0.568 a_{5, t-24}
\end{gathered}
$$

By examining the first two terms of the forecast equation, one can see that the forecast for time $t+1$ takes the NDVI value from time $t-23$ (the previous year's value at that time of month) and reduces it by the deterministic trend term, $\mu_{3}=-3.301$. This constant term is significantly different from zero $(p=0.041)$, reinforcing the conclusions drawn from the multiple testing procedure of Clements (2013). The value $\mu_{3}=-3.301$ indicates that, from year-toyear, the NDVI values are decreasing by 3.301 , on average.

The remaining terms in the forecasting equation can be attributed to the autoregressive and moving average components' past lags of Kilosa, Tanzania $\left(\boldsymbol{Z}_{3, t}\right)$ and its neighbors $\left(\boldsymbol{Z}_{1, t}, \boldsymbol{Z}_{2, t}, \boldsymbol{Z}_{4, t}, \boldsymbol{Z}_{5, t}\right)$. Out of the four neighbors, the series $\boldsymbol{Z}_{5, t}$ is more dominant than $\boldsymbol{Z}_{1, t}, \boldsymbol{Z}_{2, t}, \boldsymbol{Z}_{4, t}$, as seen by the magnitude of the AR and MA coefficients. In particular, many AR and MA components for $\boldsymbol{Z}_{4, t}$ were estimated to be extremely small. Thus, to ease interpretation, several of these small coefficients were constrained to be exactly 0 using restricted MLE's. 
This model's forecast for one year (24 lags) ahead, with the 95\% bands, is displayed in Figure 5.

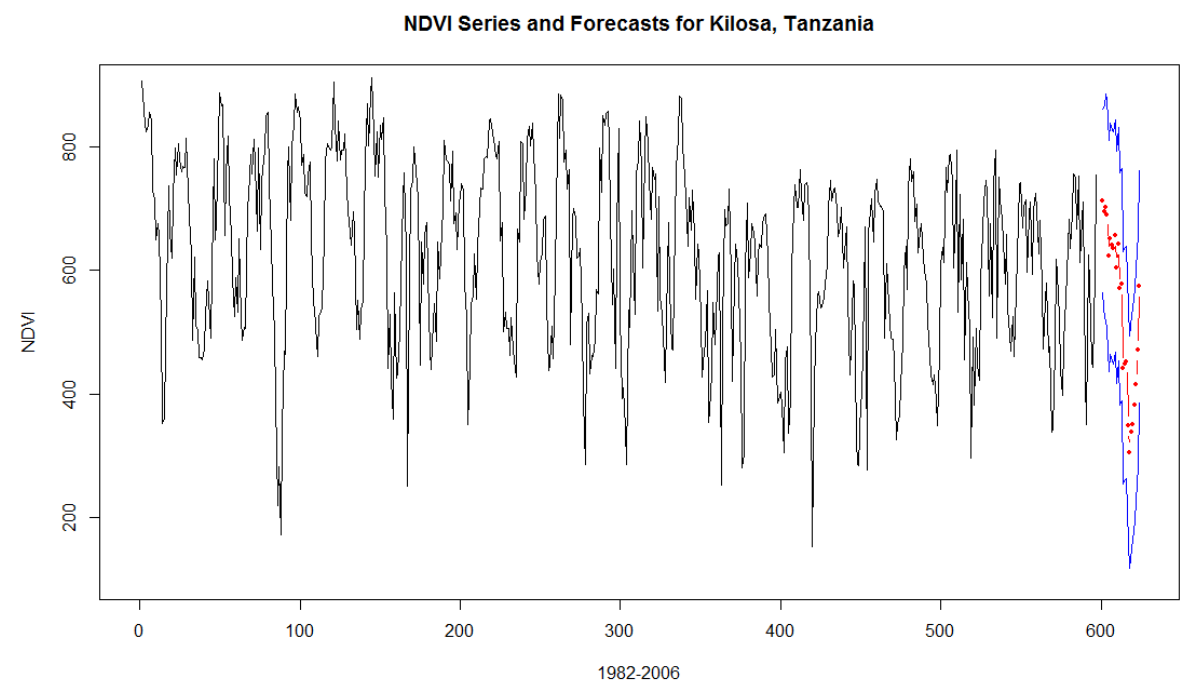

Figure 5: Kilosa's NDVI Series With One Year Forecasts And Confidence Bands

\section{SUMMARY, DISCUSSION, AND CONCLUSION}

This study focused on implementing modified spatio-temporal auto-regressive moving average (STARMA) models for sub-regions of pixel's NDVI series in the East African data. The modifications included a multiplicative component to account for the highly seasonal processes and a deterministic trend term to model the temporal trends. The authors are particularly interested in modeling and forecasting the regions in which a multiple testing procedure, designed to control an overall measure of mixed directional and non-directional false discoveries, had previously detected significant increasing or decreasing changes in the NDVI values.

Using two example pixels (Terkaka, South Sudan and Kilosa, Tanzania), we found adequate multiplicative STARMA models with significant deterministic trend terms that correspond to the trend changes detected in the multiple testing procedure. The models necessarily have a multiplicative seasonal moving-average component to account for the seasonal process.

To assess the accuracy and potential advantages of our multiplicative STARMA models, we re-estimated the models after withholding the last year of data (2006 data) and compared our forecasts with the actual observations by computing the mean square error (MSE). For Terkaka, South Sudan's NDVI series, we obtained MSE $=2,101$ and for Kilosa, Tanzania, the MSE=1,962. To get a sense of our accuracy compared to traditional models, we used univariate ARIMA models on the Terkaka and Kilosa series, also withholding 2006 data. These univariate models differ from the multiplicate STARMA models in several ways: 1) they account for the seasonal patterns only through seasonal differencing (not multiplicative AR or MA components), 2) they will not have a deterministic trend term, and 3) they will not account for any spatial dependency of neighboring pixels. As expected, the MSE using traditional ARIMA modeling was much higher in both the Terkaka series (MSE=2,251; a $7.1 \%$ increase) and the Kilosa series (MSE $=2,073$; a $5.7 \%$ increase).

In conclusion, we propose to use more sophisticated time series modeling; namely, multiplicative STARMA models with deterministic trend terms, when modeling sub-regions of pixel's NDVI series in East Africa. We have explained how to arrive at such models though model selection and estimation and demonstrated their advantages over traditional modeling. 


\section{ACKNOWLEDGEMENTS}

The NDVI dataset was collected as part of a Michigan State University research project; namely, the 'Dynamic Interactions among People, Livestock, and Savanna Ecosystems under Climate Change' project (funded by the National Science Foundation Biocomplexity of Coupled Human and Natural Systems Program, Award No. BCS/CNH 0709671). The present research of Clements and Sarkar is supported by NSF Grants DMS-1006344 and DMS-1006344 \& 1208735 .

\section{AUTHOR INFORMATION}

Dr. Clements is an Assistant Professor in the Department of Decision System Sciences at Saint Joseph's University in Philadelphia, PA. She earned her B.S. in Mathematics from Millersville University in 2008, M.S. in Statistics from Virginia Tech in 2009, and Ph.D. in Statistics from Temple University in 2013.

Dr. Sarkar is the Cyrus H. K. Curtis Professor and Chair of the Department of Statistics at Temple University in Philadelphia, PA. He has been on the faculty for over 25 years. He earned his M.S. in Statistics (1976) from Calcutta University and Ph.D. in Statistics (1982), also from Calcutta University.

Dr. Wei is Professor of Statistics at Temple University in Philadelphia, PA. He has been on the faculty since 1974. He earned his B.A. in Economics from the National Taiwan University (1966), B.A. in Mathematics from the University of Oregon (1969), and M.S. (1972) and Ph.D. (1974) in Statistics from the University of Wisconsin Madison.

\section{REFERENCES}

1. Clements, N., S. Sarkar, Z. Zhao, and D. Kim (2013), Applying Multiple Testing Procedures to Detect Changes in East African Vegetation, Annals of Applied Statistics (In Press).

2. Curran, P. (1980), Multispectral Remote Sensing of Vegetation Amount, Progress in Physical Geography, 4, 315 .

3. Davenport, M., and S. Nicholson (1993), On the Relation Between Rainfall and the Normalized Difference Vegetation Index for Diverse Vegetation Types in East Africa, International Journal of Remote Sensing, $14,2369-2389$.

4. Duveiller, G., P. Defourny, B. Desclee, and P. Mayaux (2008), Deforestation in Central Africa: Estimates at Regional, National and Landscape Levels by Advanced Processing of Systematically-Disturbed Landsat Extracts, Remote Sensing of Environment, 112, 1969-1981.

5. Hillmer, S. C., and G. C. Tiao (1979), Likelihood Function of Stationary Multiple Autoregressive Moving Average Models, Journal of the American Statistical Association, 74, 652-660.

6. Jackson, R., P. Slater, and P. Pinter (1983), Discrimination of Growth and Water Stress in Wheat by Various Vegetation indices Through Clear and Turbid Atmospheres, Remote Sensing of Environment, 15, 187.

7. Pelkey N., C. Stoner, and T. Caro (2000), Vegetation in Tanzania: Assessing Long Term Trends and Effects of Protection Using Satellite Imagery, Biological Conservation, 94, 297-309.

8. Pelkey N., C. Stoner, and T. Caro (2003), Assessing Habitat Protection Regimes in Tanzania using AVHRR NDVI Composites: Comparisons at Different Spatial Scales, International Journal of Remote Sensing, 24, 2533-2558.

9. Tiao, G., and G. Box (1981), Modeling Multiple Time Series with Applications, Journal of the American Statistical Association, 76, 802-816.

10. Tiao, G., and R. Tsay (1983), Multiple Time Series Modeling and Extended Sample Cross-Correlations, Journal of Business and Economic Statistics, 1, 43-56.

11. Tucker, C., J. Pinzon, M. Brown, D. Slayback, E. Pak, R. Mahoney, E. Vermote, and N. Saleous (2005), An Extended AVHRR 8-km NDVI Data Set Compatible with MODIS and Spot Vegetation NDVI Data, International Journal of Remote Sensing, 26, 4485-4498. 
12. Vrieling, A., K. de Beurs, and M. Brown (2008), Recent Trends in Agricultural Production of Africa Based on AVHRR NDVI Time Series, Proceedings of the SPIE conference: Remote Sensing for Agriculture, Ecosystems and Hydrology X, 71040R-71040R.

13. Wei, W. W. S. (2006), Time Series Analysis Univariate and Multivariate Methods, 2nd Edition, Pearson, Addison Wesley.

14. Wilson, G. T. (1973), The Estimation of Parameters in Multivariate Time Series Models, Journal of the Royal Statistical Society, Series B, 35, 76-85. 